\title{
Municipal Solid Waste Status in Iran; From Generation to Disposal
}

\section{Zeynab Golhosseini}

Shahid Beheshti University

Mahdi Jalili Ghazizade ( $\sim$ ma_jalili@sbu.ac.ir)

Shahid Beheshti University https://orcid.org/0000-0001-9154-2400

\section{Research Article}

Keywords: Municipal solid waste, Waste generation rate, Waste composition, Waste disposal method

Posted Date: September 27th, 2021

DOI: https://doi.org/10.21203/rs.3.rs-792222/v1

License: () (1) This work is licensed under a Creative Commons Attribution 4.0 International License. Read Full License 


\section{Abstract}

The critical step of policymaking and planning for the local governments to select appropriate waste management methods and accomplish the required programs and projects, is comprehensive information on solid waste generation. Since in the last decade there has not been a comprehensive and integrated study for identifying waste management practices at a nationwide level, so this paper presents an overview of the current solid waste generation, characteristics and disposal method covering all 31 provinces of Iran based on available information and integrated waste management plans. The results show the average of solid waste generation (municipal and rural) throughout the country is 630 grams per capita per day. Although this is 10 percent higher than its level in the past decade, it is still lower than the global average per capita (740 grams per day). Solid waste composition in Iran comprises primarily organic, with 70 percent, followed by paper and plastic that account 7 percent, make up the waste stream. In addition, on average over 90 percent of generated solid waste in Iran is still openly dumped that increase healthy and environmental risk. So it is suggested that due to obtain accurate and reliable data, a comprehensive waste monitoring plan at the national level should be defined and waste composition and generation rate determined and updated through a standard method within certain interval time. According to the data of this research, planning and financial support for source separation can decrease environmental, economic and social problems in current solid waste management systems.

\section{Introduction}

High rate of population growth and urbanization along with increasing per capita waste generation make Solid Waste Management (SWM) as a major environmental and public health concern in developing countries (Zurbrugg, 2002). Besides, challenges of SWM in developing countries is more complicated due to the legal, technical, financial, institutional, economic, and social problems (Abd Manaf et al., 2009). Adverse effects of solid waste on the environment and human health along with significant related cost imposed on the societies necessitate authorities to have a comprehensive plan on SWM. Thus, in addition to technical aspect of SWM, health and environmental issues as well as the economic and social aspects should be considered. In Iran, waste management systems are subjected to array of problems and challenges due to lack of a national strategic plan setting out a roadmap for decision makers. On the one hand, the rate of waste generation in Iran is increasing due to economic growth and urbanization, and on the other hand, changing lifestyle and consumption patterns can affect the waste composition. Drawing a roadmap for SWM systems and consequently selecting the suitable methods for storage, collection and disposal of solid waste requires a comprehensive characterization of the quantity and quality of generated waste. Although waste management plans are mostly carried out on a local or regional scale, but programming, implementation and monitoring of waste management plans at the local level should follow up large-scale policies at the national level. Therefore, waste characterization at the national level is crucial and building up a strategic plan on SWM systems is necessary. It requires having more reliable data on waste composition and quantity in the current situation and anticipating its future trend (Burnley, 2007). Actually, waste characterization at national level gives the opportunity to policymakers to allocate enough fund and infrastructure to local government, evaluate the new waste related technologies and set up a field for collaboration between local government, private section and the other stockholders.

Several studies have been carried out on the characterization of waste generated in different countries. Regarding annual reports, Municipal Solid Waste (MSW) generation in different Poland cities varies from 238 to $309 \mathrm{~kg}$ per capita. This situation is affected by a number of factors such as various methods and locations for waste sampling, several methods for field analysis and different time span for waste sampling (Den Boer et al., 2010). In another study in African countries, the waste generated in the main cities of Africa varies from 0.3 to $1.4 \mathrm{~kg}$ per day per capita with the daily average of $0.78 \mathrm{~kg} / \mathrm{per}$ capita and significant standard deviation. Comparison of the standard deviation per capita of waste generated in African cities with its corresponding value in developed countries shows that waste generation changes in developing countries are more than developed countries, which in turn makes long-term planning difficult in these countries (Achankeng, 2003). In a comprehensive research, waste management challenges were examined in more than thirty urban areas in 22 developing countries in four different continents. In this study, a combination of different methods were used to assess the factors influencing the performance of SWM in the cities. Different data about waste generation rate, collection and transportation frequency and final disposal method were given. The studied cities were a mixture of cultures and included variety of SWM systems. One of the most important outcome of this research is that the authorities should have a reliable data on the quality and quantity of MSW in order to make proper and integrated waste management strategies adapted to the requirements of the citizens considering their ability to pay for the services provided. The financial support of the central government, the interest of the municipal leaders in waste management issues, the public participation and the proper 
administration of the funds are essential for a modernized sustainable system (Guerrero et al., 2013). Zhang et al. in 2010 published a paper on SWM systems and its problems in China shows that besides urbanization, population growth and industrialization, the quantity of MSW generation has been dramatically increased in recent years. Daily per capita solid waste generation rate increased from $0.50 \mathrm{~kg}$ in 1980 to $0.98 \mathrm{~kg}$ in 2006. Currently, waste composition in China is dominated by a high organic and moisture content, since the concentration of kitchen waste in urban solid waste makes up the highest proportion about $60 \%$ of the waste stream. The total amount of MSW collected and transported was 148 million tons in 2006 , of which $91.4 \%$ was landfilled, $6.4 \%$ was incinerated and $2.2 \%$ was composted (Zhang et al., 2010). India is another country with the waste generation pattern similar to Iran. In India, per capita generation of MSW ranges from 0.2 to $0.5 \mathrm{~kg}$ per day. About $90 \%$ of MSW is disposed of unscientifically in open dumps and landfills, creating problems to public health and the environment. Such dumping has led to heavy metals rapidly leaching into the coastal waters (Sharholy et al., 2008). In 2009, a waste management study conducted in Malaysia that evaluating the amount of waste generation, characterization and management of solid waste in this country. The waste generation rate was about $0.5-0.8 \mathrm{~kg}$ per capita per day with the majority of organic waste (Manaf et al., 2009). In 2012, a comprehensive study on generation rate, composition and waste management was conducted worldwide. It shows that on average the developed countries typically generate $522-759 \mathrm{~kg}$ per capita per year, while this amount is $109-525 \mathrm{~kg}$ per capita per year in developing countries. Both in developing and developed countries, the main disposal method of municipal solid waste is landfilling, with the exception that in developed countries, landfills are in systematic manner, however in developing countries are mainly open dump (Hoornweg \& Bhada., 2012). In recent years, due to the low economic benefits of waste separation and recycling, resource recovery in the form of heat and electricity production has gained favor. in Asia and other developing countries, the composition of the generated MSW is around $40-80 \%$ of MSW comprises organic waste, while in Europe and developed American continents an average of $30-40 \%$ of MSW consist of organic waste (Karak et al., 2012).

Despite the importance of waste management issue, in Iran there has been no integrated and comprehensive data about solid waste. Hasanwand et al. (2008) published the latest research on the solid waste identification carried out by the Ministry of the Interior in 2004. This study reveals that, average of municipal solid waste generation in the country was $0.64 \mathrm{~kg}$ per capita per day and the waste composition was $72.04 \%$ organic material, $6.43 \%$ paper and cardboard, $7.77 \%$ plastic, $2.52 \%$ metals, $1.14 \%$ rubber, $2.86 \%$ textiles, $2.03 \%$ glass, $1.10 \%$ wood and $4.11 \%$ other materials. Based on the results of this research, 10.3 million tons of MSW was generated in 2004 , where $6 \%$ was recycled, $10 \%$ was composted, and $84 \%$ was mostly unprocessed dumped (Hassanvand et al., 2008). Since MSW quantity and quality can affect by economic development and degree of industrialization, so it is expected that the quantity and composition of generated MSW in Iran have been significantly changed through last decade. However, integrated studies on the identification of Iran's generated waste have not been carried out since 2004. The lack of documented information on the waste characterization in Iran has even reflected in international reports in the way that the World Bank reports on the world MSW statistics (published in 2012) has used Damghani et al. (2008) papers to present the status of waste generation in Iran (Hoornweg et al., 2012). However, the information provided by Damghani et al. (2008) is merely related to the Tehran city and it is impossible to generalize mentioned information to the whole country. In the latest edition of the World Bank report (published in 2018), an informal report by Abedini (2017) was used as the source of information on Iran SWM (Kaza et al., 2018). Therefore, it is necessary to examine SWM conditions in in different parts of the country to produce more reliable data on waste generation trends. On the other hand, according to the Waste Management Act (approved in 2004), all cities with a population of more than one million inhabitants, must have provided a comprehensive waste management plan by the end of 2011 (Waste Management Act, 2004). Therefore, this paper presents an overview of current status of MSW generation and disposal methods by collecting data from different cities of the country reported in various sources.

\section{Background Information}

Iran is located in the southwest of Asia and in the Middle East with 1648195 square kilometers in size and population of 79926270 inhabitants (based on the census of 2016). Based on the latest divisions of the country in 2014, Iran consists of 31 provinces. The capital, the largest city and cultural, economic, political and administrative center of Iran, is Tehran. Different cultures and weather conditions make adoption of a waste management plan at national level very complicated and it will be impossible in the absence of sufficient information on waste characterization produced in various parts of the country. The present study has collected and analyzed data of wastes generated in the capital of all provinces. To this end, the existing reports were used for cities with comprehensive waste management plan and available information, and in the absence of such data, information have been 
collected from other reliable sources and authorities. Table 1 shows general data of provincial capitals in Iran as well as sources used for identifying wastes produced in each region. 
Table 1

Provincial capitals information and data collection resources

\begin{tabular}{|c|c|c|c|c|c|c|c|}
\hline \multirow[t]{2}{*}{ Province } & \multirow[t]{2}{*}{ Capital } & \multicolumn{4}{|c|}{$\begin{array}{l}\text { General information of capital (National } \\
\text { statistical yearbook, 2016) }\end{array}$} & \multirow[t]{2}{*}{ Year } & \multirow[t]{2}{*}{ Reference } \\
\hline & & $\begin{array}{l}\text { Area } \\
\left(\mathrm{Km}^{2}\right)\end{array}$ & $\begin{array}{l}\text { Population } \\
\text { (millions) }\end{array}$ & $\begin{array}{l}\text { Density } \\
\text { (per } \\
\mathrm{Km}^{2} \text { ) }\end{array}$ & $\begin{array}{l}\text { Annual } \\
\text { population } \\
\text { growth rate } \\
(\%)\end{array}$ & & \\
\hline Alborz & Karaj & 162 & 1.94 & 11978 & -0.27 & 2013 & Hosseini et al., 2013 \\
\hline Ardabil & Ardabil & 111 & 0.53 & 4796 & 1.86 & 2014 & Naseri et al., 2015 \\
\hline Bushehr & Bushehr & 70 & 0.27 & 3908 & 2.74 & 2007 & Ramavandi et al., 2014 \\
\hline $\begin{array}{l}\text { Chaharmahal } \\
\text { and Bakhtiari }\end{array}$ & Shahrekord & 45 & 0.28 & 6404 & 3.57 & 2012 & $\begin{array}{l}\text { Sanatpouyan sabz kavir Cons } \\
\text { Eng. Co., } 2012\end{array}$ \\
\hline $\begin{array}{l}\text { East } \\
\text { Azerbaijan }\end{array}$ & Tabriz & 324 & 1.62 & 7780 & 0.83 & 2006 & $\begin{array}{l}\text { Sabz Andish Payesh Cons. } \\
\text { Eng. Co., 2010a }\end{array}$ \\
\hline Esfahan & Esfahan & 551 & 2.11 & 3834 & 2.23 & 2014 & Isfahan Municipality, 2014 \\
\hline Fars & Shiraz & 224 & 1.71 & 7647 & 1.39 & 2008 & Shiraz University, 2015 \\
\hline Gilan & Rasht & 60 & 0.74 & 12478 & 1.22 & 2002 & $\begin{array}{l}\text { Ravanbakhsh et al., 2008; } \\
\text { Mohammadi Golrang et al., } \\
2011\end{array}$ \\
\hline Golestan & Gorgan & 40 & 0.36 & 9142 & 1.25 & 2010 & $\begin{array}{l}\text { SabzAndish Payesh Cons. } \\
\text { Eng. Co., 2010b }\end{array}$ \\
\hline Hamedan & Hamedan & 73.6 & 0.57 & 7910 & 1.06 & 2005 & Maleki \& Omrani, 2002 \\
\hline Hormozgan & $\begin{array}{l}\text { Bandar } \\
\text { Abbas }\end{array}$ & 80 & 0.54 & 6790 & 3.86 & 2007 & $\begin{array}{l}\text { Fanavari Behmand Cons. Eng. } \\
\text { Co., } 2007\end{array}$ \\
\hline Ilam & llam & 60 & 1.99 & 3316 & 2.41 & 2014 & $\begin{array}{l}\text { Zistaza mohit Cons. Eng. Co., } \\
2014\end{array}$ \\
\hline Kerman & Kerman & 185 & 0.63 & 3417 & 0.12 & 2012 & $\begin{array}{l}\text { Tosiepaydar shomalgan } \\
\text { Cons.Eng. Co., } 2006\end{array}$ \\
\hline Kermanshah & Kermanshah & 96 & 0.95 & 9919 & 2.14 & 2011 & $\begin{array}{l}\text { Soleimanzadeh et al., 2013; } \\
\text { Omrani. } 2007\end{array}$ \\
\hline $\begin{array}{l}\text { Kohgiluyeh } \\
\text { and Boyer- } \\
\text { Ahmad }\end{array}$ & Yasuj & 30 & 0.13 & 4484 & 4.39 & 2012 & $\begin{array}{l}\text { Yasouj University of Medical } \\
\text { Science, } 2012\end{array}$ \\
\hline Khuzestan & Ahvaz & 185 & 1.19 & 6445 & 1.27 & 2007 & $\begin{array}{l}\text { Sazab pardazan Cons. Eng. } \\
\text { Co., } 2006\end{array}$ \\
\hline Kurdistan & Sanandaj & 31 & 0.41 & 13357 & 1.99 & 2006 & Heydari et al., 2013 \\
\hline Lorestan & Khorramabad & 63 & 0.37 & 5927 & 1.4 & 2008 & $\begin{array}{l}\text { SabzAndish Payesh Cons. } \\
\text { Eng. Co., 2010c }\end{array}$ \\
\hline Markazi & Arak & 60 & 0.53 & 8859 & -0.19 & 2008 & $\begin{array}{l}\text { Pars amayesh Cons. Eng. Co., } \\
2010\end{array}$ \\
\hline Mazandaran & Sari & 30 & 0.31 & 10400 & 0.88 & 2008 & $\begin{array}{l}\text { Mazandaran Recycling } \\
\text { Institute, } 2010\end{array}$ \\
\hline $\begin{array}{l}\text { North } \\
\text { Khorasan }\end{array}$ & Bojnord & 36 & 0.23 & 6495 & 2.76 & 2015 & $\begin{array}{l}\text { Dadsetan et al., } \\
\text { 2017; Hajinezhad et al., } 2015\end{array}$ \\
\hline Qazvin & Qazvin & 65 & 0.48 & 7470 & 1.08 & 2008 & $\begin{array}{l}\text { Environmental Research } \\
\text { Center of Tehran University of } \\
\text { Medical Sciences, } 2010\end{array}$ \\
\hline
\end{tabular}




\begin{tabular}{|c|c|c|c|c|c|c|c|}
\hline \multirow[t]{2}{*}{ Province } & \multirow[t]{2}{*}{ Capital } & \multicolumn{4}{|c|}{$\begin{array}{l}\text { General information of capital (National } \\
\text { statistical yearbook, 2016) }\end{array}$} & \multirow[t]{2}{*}{ Year } & \multirow[t]{2}{*}{ Reference } \\
\hline & & $\begin{array}{l}\text { Area } \\
\left(\mathrm{Km}^{2}\right)\end{array}$ & $\begin{array}{l}\text { Population } \\
\text { (millions) }\end{array}$ & $\begin{array}{l}\text { Density } \\
\text { (per } \\
\left.\mathrm{Km}^{2}\right)\end{array}$ & $\begin{array}{l}\text { Annual } \\
\text { population } \\
\text { growth rate } \\
(\%)\end{array}$ & & \\
\hline Qom & Qom & 123 & 1.22 & 7257 & 2.26 & 2011 & Azari et al., 2011 \\
\hline $\begin{array}{l}\text { Razavi } \\
\text { Khorasan }\end{array}$ & Mashhad & 328 & 3.01 & 9183 & 1.76 & 2012 & $\begin{array}{l}\text { Rastegar et al., 2015; Shokooh } \\
\text { et al., } 2013\end{array}$ \\
\hline Semnan & Semnan & 40 & 0.18 & 4628 & 3.79 & 2016 & $\begin{array}{l}\text { Semnan University } \\
\text { Environmental Research } \\
\text { Institute, } 2016\end{array}$ \\
\hline $\begin{array}{l}\text { Sistan and } \\
\text { Baluchestan }\end{array}$ & Zahedan & 90 & 0.59 & 6588 & 0.94 & 2010 & $\begin{array}{l}\text { Safari et al., 2011; Jalilvand et } \\
\text { al., } 2013\end{array}$ \\
\hline $\begin{array}{l}\text { South } \\
\text { Khorasan }\end{array}$ & Birjand & 30 & 0.20 & 6787 & 2.72 & 2010 & Valizadeh et al., 2015 \\
\hline Tehran & Tehran & 750 & 8.69 & 11591 & 1.28 & 2020 & $\begin{array}{l}\text { SabzAndish Payesh Cons. } \\
\text { Eng. Co., } 2020\end{array}$ \\
\hline $\begin{array}{l}\text { West } \\
\text { Azerbaijan }\end{array}$ & Urmia & 105 & 0.75 & 7117 & 1.97 & 2013 & Taraghi Nazlou et al., 2015 \\
\hline Yazd & Yazd & 99.5 & 0.61 & 6145 & 1.72 & 2012 & $\begin{array}{l}\text { Idehpardazan tousei Cons. } \\
\text { Eng. Co., } 2012\end{array}$ \\
\hline Zanjan & Zanjan & 150 & 0.43 & 2889 & 2.17 & 2009 & Taghvayi et al., 2012 \\
\hline
\end{tabular}

\section{Waste Generation}

MSW generation rate is effected by economic situations, cultural conditions, eating habits, and local climate. Therefore, the rate of waste generation in different parts of the country can be significantly different. Table 2 shows the per capita waste generation rate and the total amount of waste generation in municipal and rural area of all provinces of Iran. MSW generation rate is averagely 0.70 $\mathrm{kg} / \mathrm{ca} /$ day and the average of Rural Solid Waste (RSW) generation rate is $0.44 \mathrm{~kg} / \mathrm{ca} /$ day.

Accordingly, the annual waste generation rate in the country (municipal and rural) is $0.63 \mathrm{~kg} / \mathrm{capita} / \mathrm{day}$, the largest per capita solid waste generation rates are found in Gilan and afterwards in Khuzestan with an average of 0.93 and $0.83 \mathrm{~kg} / \mathrm{person} / \mathrm{day}$. Sistan and Baluchestan with an average of $0.32 \mathrm{~kg} / \mathrm{capita} /$ day has the lowest waste generation among the provinces. Waste generation rate in Tehran, the capital of Iran, is $0.76 \mathrm{~kg} / \mathrm{capita} /$ day. Figure 1 illustrate waste generation per capita by province, indicating average daily per capita of waste generated within Iran. 
Table 2

Municipal and rural solid waste generation rate in Iran

\begin{tabular}{|c|c|c|c|c|c|c|c|}
\hline Province & $\begin{array}{l}\text { Total } \\
\text { population } \\
\text { (millions) }\end{array}$ & $\begin{array}{l}\text { Urban } \\
\text { population } \\
\text { (millions) }\end{array}$ & $\begin{array}{l}\text { MSW } \\
\text { generation } \\
\text { (tonnes/day) }\end{array}$ & $\begin{array}{l}\text { MSW } \\
\text { generation rate } \\
\text { (kg/capita/day) }\end{array}$ & $\begin{array}{l}\text { Rural } \\
\text { population } \\
\text { (millions) }\end{array}$ & $\begin{array}{l}\text { RSW } \\
\text { generation } \\
\text { (tonnes/day) }\end{array}$ & $\begin{array}{l}\text { RSW } \\
\text { generation rate } \\
\text { (kg/capita/day) }\end{array}$ \\
\hline Alborz & 2.71 & 2.50 & 1733788 & 0.69 & 0.19 & 69882 & 0.35 \\
\hline Ardabil & 1.27 & 0.86 & 536941 & 0.62 & 0.40 & 133447 & 0.33 \\
\hline Bushehr & 1.16 & 0.83 & 543371 & 0.65 & 0.32 & 208582 & 0.63 \\
\hline $\begin{array}{l}\text { Chaharmahal } \\
\text { and Bakhtiari }\end{array}$ & 0.94 & 0.60 & 206531 & 0.34 & 0.34 & 177306 & 0.52 \\
\hline $\begin{array}{l}\text { East } \\
\text { Azerbaijan }\end{array}$ & 3.90 & 2.80 & 1994691 & 0.71 & 1.10 & 297062 & 0.27 \\
\hline Esfahan & 5.12 & 4.50 & 2433947 & 0.54 & 0.61 & 307998 & 0.50 \\
\hline Fars & 4.85 & 3.40 & 2687323 & 0.79 & 1.44 & 533452 & 0.36 \\
\hline Gilan & 2.53 & 1.60 & 1603026 & 1.00 & 0.92 & 760689 & 0.82 \\
\hline Golestan & 1.86 & 0.99 & 615290 & 0.61 & 0.87 & 471530 & 0.54 \\
\hline Hamedan & 1.73 & 1.09 & 822913 & 0.75 & 0.64 & 277560 & 0.43 \\
\hline Hormozgan & 1.77 & 0.97 & 758021 & 0.78 & 0.80 & 466664 & 0.58 \\
\hline Ilam & 0.58 & 0.39 & 237158 & 0.60 & 0.18 & 57687 & 0.31 \\
\hline Kerman & 3.16 & 1.85 & 1263839 & 0.68 & 1.30 & 382696 & 0.29 \\
\hline Kermanshah & 1.95 & 1.46 & 851797 & 0.58 & 0.48 & 209494 & 0.43 \\
\hline $\begin{array}{l}\text { Kohgiluyeh } \\
\text { and Boyer- } \\
\text { Ahmad }\end{array}$ & 0.71 & 0.39 & 270273 & 0.68 & 0.31 & 97833 & 0.31 \\
\hline Khuzestan & 4.71 & 3.55 & 3554205 & 1.00 & 1.15 & 381580 & 0.33 \\
\hline Kurdistan & 1.60 & 1.13 & 703222 & 0.62 & 0.46 & 180950 & 0.38 \\
\hline Lorestan & 1.76 & 1.13 & 703643 & 0.62 & 0.62 & 277829 & 0.44 \\
\hline Markazi & 1.42 & 1.09 & 989788 & 0.90 & 0.32 & 209366 & 0.63 \\
\hline Mazandaran & 3.28 & 1.89 & 1713206 & 0.90 & 1.38 & 959350 & 0.69 \\
\hline $\begin{array}{l}\text { North } \\
\text { Khorasan }\end{array}$ & 0.86 & 0.48 & 348729 & 0.72 & 0.37 & 185207 & 0.48 \\
\hline Qazvin & 1.27 & 0.95 & 685547 & 0.72 & 0.32 & 210656 & 0.65 \\
\hline Qom & 1.29 & 1.22 & 811776 & 0.66 & 0.06 & 29415 & 0.47 \\
\hline $\begin{array}{l}\text { Razavi } \\
\text { Khorasan }\end{array}$ & 6.43 & 4.70 & 2350462 & 0.50 & 1.73 & 813048 & 0.46 \\
\hline Semnan & 0.70 & 0.56 & 375536 & 0.67 & 0.14 & 60006 & 0.42 \\
\hline $\begin{array}{l}\text { Sistan and } \\
\text { Baluchestan }\end{array}$ & 2.77 & 1.34 & 497888 & 0.37 & 1.42 & 414518 & 0.29 \\
\hline $\begin{array}{l}\text { South } \\
\text { Khorasan }\end{array}$ & 0.76 & 0.45 & 294988 & 0.65 & 0.31 & 145248 & 0.46 \\
\hline Tehran & 13.26 & 12.42 & 9463695 & 0.76 & 0.81 & 358779 & 0.44 \\
\hline
\end{tabular}




\begin{tabular}{|llllllll|}
\hline Province & $\begin{array}{l}\text { Total } \\
\text { population } \\
\text { (millions) }\end{array}$ & $\begin{array}{l}\text { Urban } \\
\text { population } \\
\text { (millions) }\end{array}$ & $\begin{array}{l}\text { MSW } \\
\text { generation } \\
\text { tonnes/day) }\end{array}$ & $\begin{array}{l}\text { MSW } \\
\text { generation rate } \\
\text { (kg/capita/day) }\end{array}$ & $\begin{array}{l}\text { Rural } \\
\text { population } \\
\text { (millions) }\end{array}$ & $\begin{array}{l}\text { RSW } \\
\text { generation } \\
\text { (tonnes/day) }\end{array}$ & $\begin{array}{l}\text { RSW } \\
\text { generation rate } \\
\text { (kg/capita/day) }\end{array}$ \\
\hline $\begin{array}{l}\text { West } \\
\text { Azerbaijan }\end{array}$ & 3.26 & 2.13 & 1281722 & 0.60 & 1.12 & 395156 & 0.35 \\
\hline Yazd & 1.13 & 0.97 & 582813 & 0.60 & 0.16 & 54166 & 0.32 \\
\hline Zanjan & 1.05 & 0.71 & 490712 & 0.69 & 0.34 & 179721 & 0.51 \\
\hline Annual & $\mathbf{7 9 . 9 2}$ & $\mathbf{5 9 . 1 4}$ & $\mathbf{4 1 4 0 6 8 4 1}$ & $\mathbf{0 . 7 0}$ & $\mathbf{2 0 . 7 7}$ & $\mathbf{9 3 0 6 8 7 8}$ & $\mathbf{0 . 4 4}$ \\
\hline
\end{tabular}

According to the latest report of World Bank, the average of MSW generation in 217 countries is $0.74 \mathrm{~kg} / \mathrm{capita} /$ day (Kaza et al., 2018). Income level and location are two important criteria used to compare MSW generation in Iran with the corresponding global values. Iran is categorized as an Upper Middle Income (UMI) and the waste generation rate in these countries is averagely 0.69 $\mathrm{kg} /$ person/day. On the other hand, per capita waste production in the Middle East and North Africa (MENA) region is $0.81 \mathrm{~kg}$ per day. Therefore, the average per capita waste generation in Iran is less than the corresponding value in countries where the economic and regional view is similar to Iran.

\section{Msw Composition}

Waste composition is one of the most important parameter considered for selection suitable methods for collection, processing, transportation and final disposal. Different factors such as income level, consumption pattern, geographic location, source of energy and climate influence on waste composition. Investigate waste composition allows local governments to select appropriate management methods and treatment for MSW.

Determination of the waste composition is usually done by the standard method D5231 ASTM. Throughout the documentation for MSW composition, MSW is classified as organic material (including vegetables, food, and garden waste), paper and cardboard (including paper, wrapper and packaging paper), plastics (including plastic bags, plastic bottles, and packaging material), glass/ceramics (including glass bottles, broken glass, pottery items and earthen pot), metals (cables, foils, ferrous and nonferrous material), and others (including textiles) (ASTM D5231). Table 3 shows waste composition in different cities of Iran. In general waste composition in Iran is dominated by high organic and moisture content. Among different composition in MSW, organic material contributes a higher percentage, which was $69.8 \%$. At the next steps, plastic with $7.8 \%$ and paper and cardboard with $7.4 \%$ comprise the highest amount. Glass and metal with the same percentage $(2 \%)$ have the lowest share among the waste composition in Iran. 
Table 3

MSW composition in provincial capitals in Iran

\begin{tabular}{|c|c|c|c|c|c|c|c|c|}
\hline City & Year & $\begin{array}{l}\text { Urban Population } \\
\text { (millions) }\end{array}$ & $\begin{array}{l}\text { Organic } \\
(\%)\end{array}$ & $\begin{array}{l}\text { Paper \& } \\
\text { Cardboard (\%) }\end{array}$ & $\begin{array}{l}\text { Plastics } \\
(\%)\end{array}$ & $\begin{array}{l}\text { Glass } \\
(\%)\end{array}$ & $\begin{array}{l}\text { Metals } \\
(\%)\end{array}$ & $\begin{array}{l}\text { Other } \\
\text { (\%) }\end{array}$ \\
\hline Ahvaz & 2007 & 1.14 & 62.1 & 10.5 & 12.1 & 3.3 & 1.8 & 10.3 \\
\hline Arak & 2006 & 0.44 & 66.8 & 6.8 & 2.9 & 2.4 & 1.1 & 19.9 \\
\hline Ardabil & 2014 & 0.48 & 75.2 & 4.2 & 8.7 & 2.4 & 1.7 & 7.8 \\
\hline $\begin{array}{l}\text { Bandar } \\
\text { Abbas }\end{array}$ & 2006 & 0.37 & 76.1 & 6.5 & 7.6 & 2.4 & 2.5 & 4.9 \\
\hline Birjand & 2010 & 0.18 & 77.0 & 7.4 & 5.6 & 2.2 & 1.5 & 6.4 \\
\hline Bojnord & 2015 & 0.20 & 77.5 & 5.7 & 5.5 & 1.0 & 2.1 & 8.1 \\
\hline Bushehr & 2012 & 0.20 & 70.0 & 6.9 & 8.3 & 2.6 & 4.2 & 8.0 \\
\hline Esfahan & 2008 & 1.60 & 79.6 & 3.9 & 8.7 & 1.0 & 0.5 & 6.3 \\
\hline Gorgan & 2008 & 0.27 & 71.0 & 10.0 & 7.0 & 2.3 & 1.3 & 8.4 \\
\hline Hamedan & 2007 & 0.49 & 78.8 & 3.9 & 5.5 & 1.2 & 1.6 & 9.0 \\
\hline Ilam & 2006 & 0.17 & 74.1 & 5.8 & 7.8 & 2.0 & 1.8 & 8.5 \\
\hline Karaj & 2013 & 1.61 & 78.4 & 5.9 & 6.1 & 1.6 & 1.5 & 6.5 \\
\hline Kerman & 2009 & 0.53 & 72.3 & 8.7 & 8.9 & 3.0 & 2.1 & 5.0 \\
\hline Kermanshah & 2013 & 1.94 & 66.6 & 7.2 & 9.8 & 1.3 & 1.1 & 14.0 \\
\hline Khorramabad & 2008 & 0.34 & 86.6 & 5.1 & 5.2 & 0.3 & 0.9 & 1.8 \\
\hline Mashhad & 2012 & 2.74 & 10.8 & 46.7 & 9.7 & 5.0 & 6.8 & 21.0 \\
\hline Qazvin & 2008 & 0.36 & 79.8 & 7.8 & 5.1 & 1.3 & 1.0 & 5.1 \\
\hline Qom & 2011 & 1.07 & 67.0 & 4.0 & 9.0 & 2.0 & 2.0 & 16.0 \\
\hline Rasht & 2009 & 2.45 & 65.3 & 8.8 & 11.0 & 1.7 & 1.2 & 12.1 \\
\hline Sanandaj & 2006 & 0.37 & 71.0 & 6.0 & 8.0 & 2.0 & 1.0 & 12.0 \\
\hline Sari & 2008 & 0.26 & 76.0 & 8.0 & 8.4 & 1.1 & 1.3 & 5.2 \\
\hline Semnan & 2011 & 0.15 & 72.2 & 7.6 & 7.1 & 2.6 & 2.1 & 8.4 \\
\hline Shahrekord & 2017 & 0.29 & 75.6 & 7.9 & 4.7 & 4 & 2.4 & 5.4 \\
\hline Shiraz & 2008 & 1.22 & 66.3 & 5.4 & 10.7 & 2.4 & 1.8 & 13.5 \\
\hline Tabriz & 2006 & 1.30 & 69.4 & 6.4 & 3.1 & 1.7 & 1.0 & 18.4 \\
\hline Tehran & 2020 & 8.94 & 58.0 & 9.0 & 20.0 & 3.0 & 1.0 & 9.0 \\
\hline Urmia & 2013 & 0.71 & 75.2 & 3.8 & 9.0 & 2.4 & 1.7 & 7.9 \\
\hline Yasuj & 2010 & 0.11 & 76.6 & 4.8 & 7.9 & 2.4 & 1.4 & 6.9 \\
\hline Yazd & 2009 & 0.48 & 66.8 & 5.0 & 8.5 & 2.1 & 1.6 & 16.0 \\
\hline Zahedan & 2010 & 0.46 & 48.2 & 2.1 & 2.7 & 2.0 & 2.4 & 42.7 \\
\hline Zanjan & 2009 & 0.35 & 74.6 & 5.0 & 6.3 & 2.0 & 2.5 & 9.6 \\
\hline
\end{tabular}


Figure 2 illustrates the difference in waste composition in Iran with the corresponding global values. While the average production of organic wastes in the world is $44 \%$, the share of organic wastes in the MSW composition in Iran is about 1.6 times the global average. The amount of organic waste in UMI and MENA countries is about $54 \%$ and $58 \%$ respectively. However, Iran is among the UMI countries, but the pattern of consumption in this country is almost similar to low-income countries. In addition, by comparing the average of the waste composition of Iran with the countries of the MENA region, the amount of organic waste production is higher than the countries of this region. The average production of paper waste in the countries of this region is about $13.2 \%$ and the average production of plastic waste is $11.7 \%$ but in Iran, these two elements are close together and about $7 \%$. World Bank reports on worldwide waste composition shows that the amount of plastic in generated waste has increased from 10-12\% through 5 years (Hoornweg et al., 2012; Kaza et al., 2018).

\section{Final Disposal}

Table 4 shows that most of the disposal sites in the studied cities are open dumps. In this method waste disposed in open land out of the city. In controlled landfill, waste is covered by a layer of soil for avoiding waste displacement. Landfills have very limited contribution in energy recovery and recycling from waste stream, which can be affected by the lack of source separation, adequate structure and poor waste management at various steps of the waste management system. There is no accurate information about disposal method in developing countries but generally rely on open dump. In high-income countries, the most commonly method for MSW management is landfill and recycling. According to the global average, solid waste is almost disposed in landfills, followed by compost, recycle and incineration. 
Table 4

Final disposal methods of MSW in provincial capitals in Iran

\begin{tabular}{|c|c|c|c|c|c|c|c|}
\hline City & Year & Open Dump (\%) & Landfills (\%) & Compost (\%) & Recycled (\%) & Incineration (\%) & Other (\%) \\
\hline Ahvaz & 2007 & 100.0 & 0.0 & 0.0 & 0.0 & 0.0 & 0.0 \\
\hline Arak & 2006 & 100.0 & 0.0 & 0.0 & 0.0 & 0.0 & 0.0 \\
\hline Ardabil & 2014 & 100.0 & 0.0 & 0.0 & 0.0 & 0.0 & 0.0 \\
\hline Bandar Abbas & 2006 & 100.0 & 0.0 & 0.0 & 0.0 & 0.0 & 0.0 \\
\hline Birjand & 2006 & 100.0 & 0.0 & 0.0 & 0.0 & 0.0 & 0.0 \\
\hline Bojnord & 2007 & 100.0 & 0.0 & 0.0 & 0.0 & 0.0 & 0.0 \\
\hline Bushehr & 2012 & 98.0 & 0.0 & 0.0 & 2.0 & 0.0 & 0.0 \\
\hline Esfahan & 2008 & 0.0 & 50.3 & 47.0 & 2.7 & 0.0 & 0.0 \\
\hline Gorgan & 2008 & 0.0 & 90.0 & 10.0 & 0.0 & 0.0 & 0.0 \\
\hline Hamedan & 2007 & 0.0 & 99.0 & 0.0 & 1.0 & 0.0 & 0.0 \\
\hline Ilam & 2006 & 100.0 & 0.0 & 0.0 & 0.0 & 0.0 & 0.0 \\
\hline Karaj & 2013 & 100.0 & 0.0 & 0.0 & 0.0 & 0.0 & 0.0 \\
\hline Kerman & 2009 & 0.0 & 85.0 & 15.0 & 0.0 & 0.0 & 0.0 \\
\hline Kermanshah & 2006 & 0.0 & 0.0 & 75.0 & 7.0 & 0.0 & 20.0 \\
\hline Khorramabad & 2008 & 100.0 & 0.0 & 0.0 & 0.0 & 0.0 & 0.0 \\
\hline Mashhad & 2006 & 80.0 & 0.0 & 20.0 & 0.0 & 0.0 & 0.0 \\
\hline Qazvin & 2008 & 0.0 & 95.5 & 0.0 & 0.5 & 0.0 & 0.0 \\
\hline Qom & 2011 & 95.0 & 0.0 & 0.0 & 5.0 & 0.0 & 0.0 \\
\hline Rasht & 2002 & 66.6 & 0.0 & 25.0 & 8.3 & 0.0 & 0.0 \\
\hline Sanandaj & 2006 & 36.0 & 0.0 & 0.0 & 64.0 & 0.0 & 0.0 \\
\hline Sari & 2008 & 100.0 & 0.0 & 0.0 & 0.0 & 0.0 & 0.0 \\
\hline Semnan & 2011 & 100.0 & 0.0 & 0.0 & 0.0 & 0.0 & 0.0 \\
\hline Shahrekord & 2017 & 100.0 & 0.0 & 0.0 & 0.0 & 0.0 & 0.0 \\
\hline Shiraz & 2008 & 0.0 & 98.8 & 0.7 & 0.5 & 0.0 & 0.0 \\
\hline Tabriz & 2006 & 99.0 & 0.0 & 0.0 & 1.0 & 0.0 & 0.0 \\
\hline Tehran & 2020 & 0.0 & 45.0 & 15.0 & 40.0 & 0.0 & 0.0 \\
\hline Urmia & 2013 & 100.0 & 0.0 & 0.0 & 0.0 & 0.0 & 0.0 \\
\hline Yasuj & 2010 & 0.0 & 100.0 & 0.0 & 0.0 & 0.0 & 0.0 \\
\hline Yazd & 2009 & 100.0 & 0.0 & 0.0 & 0.0 & 0.0 & 0.0 \\
\hline Zahedan & 2012 & 100.0 & 0.0 & 0.0 & 0.0 & 0.0 & 0.0 \\
\hline Zanjan & 2009 & 50.0 & 30.0 & 20.0 & 0.0 & 0.0 & 0.0 \\
\hline
\end{tabular}

Figure 3 compares the waste disposal methods in Iran with the global average, UMI and MENA countries. As Shown in Fig. 6, different kind of waste landfilling (i.e. open dump, controlled landfill, engineered landfill and etc.) is prevalent method in Iran, Mena and UMI countries. However, in UMI countries sanitary landfill is more common, while in Iran and Mena countries, open dumping is the most popular method for waste disposal. 


\section{Conclusion}

The most important prerequisite to provide long-term waste management plans at national level is to identify the quality and quantity of generated waste and predict waste changes trend. No comprehensive and integrated action has been made to characterize solid waste generation in Iran within the past decade. Therefore, in this study, a thorough analysis on waste management conditions in the country was done using available and reliable data in order to identify the strong and weak points of the existing system and set a platform for developing a long-term comprehensive plan. Thus, characterization and analysis of three important factors of waste specification (i.e. quality, quantity and disposal method) has been taken into account in the provincial cities.

In terms of quantity of waste generation, results show that the per capita waste generation in Iran is not only under the global average, but even less than Iran's peer countries from geographical and economic point of view. The data indicates that although waste reduction programs should be pursued, but the focus should be on promoting waste separation plans along with encouraging disposal methods with the approach of maximum material and energy recovery.

The study of waste composition in the Iran's cities indicated that major part of the citizens' waste composition (more than 70\%) is putrescible waste. Although, affordable food products as compared with other goods and the unique consumption pattern in Iran (as compared with other countries) are the main reasons of large amount of organic wastes. In addition, informal recycling system in most Iran's cities is also effective in this regard. In the other word, lack of proper supervision on waste storage and collection process results in dramatic increasing scavengers which in turn leads to removal of major part of dry recyclable trash (e.g. plastics, paper and cardboard, metals and etc.). Therefore, besides planning for regular waste sampling and analysis, the strong recommendation is to organize informal recycling systems to obtain real statistics of generated waste quantity and quality.

With respect to waste disposal methods, most of measures have been taken within the past decade in Iran and results show that open dump is still the prevailing method in urban wastes disposal throughout the country and using other standard methods (i.e. sanitary landfill, recycling, incineration and etc.) is much less than the global average. This issue is basically related to lack of national standard on avoidance of open dumping as well as weakness of supreme Iran's environmental organizations who have no sound perception of nationwide policy on solid waste disposal. In addition, the blurred place of consulting engineers in decisionmaking system in the waste management cycle has made the concept of choosing best disposal method with respect to the waste specifications and the exclusive condition of each city a missing link in the waste management comprehensive plans.

Although, the present paper provides a general framework of waste generation and disposal methods throughout the country by collecting, classifying and analyzing available data, we need to adopt a comprehensive monitoring plan in waste management system. A systematic data gathering platform on quality and quantity of generated waste should be designed based on standard sampling methods to produce reliable information within specific time intervals for all Iran's cities in order to adopt mid-term and long-term strategic plans through identifying potentials and review of the process of changes.

\section{Declarations}

- Ethical Approval: Not applicable

- Consent to Participate: Not applicable

- Authors Contributions: Mahdi Jalili Ghazizade performed conceptualization and writing - review and editing. Zeynab Golhosseini performed data collection and writing - original draft preparation.

- Competing Interests: The authors declare that they have no competing interests

- Founding: No funds, grants, or other support was received. The authors have no financial or proprietary interests in any material discussed in this article.

- Availability of data and materials: The datasets used during the current study are available from the corresponding author on reasonable request.

- Acknowledgements: Not applicable 


\section{References}

1. Abd Manaf, L., Abu Sameh, M. A., Mohd Zukki, N. I. (2009). Municipal Solid Waste Management in Malaysia: Practices and Challenges. Waste Management. 29, 2902-2906.

2. Achankeng, E. (2003). Globalization, urbanization and municipal solid waste management in Africa. In Proceedings of the African Studies Association of Australasia and the Pacific 26th annual conference.

3. ASTM D5231-92(2016), Standard Test Method for Determination of the Composition of Unprocessed Municipal Solid Waste, ASTM International, West Conshohocken, PA, 2016, www.astm.org

4. Azari, A., Farzadkia, M., Rastegar, A., \& Ahmadi, E. (2011). A 20-Year Perspective on Dry Waste Recycling in Qom and Its Economic Profits. Journal of Sabzevar University of Medical Sciences. Volume 20 / Number 24/1392

5. Burnley, S. J. (2007). A review of municipal solid waste composition in the United Kingdom. Waste management, 27(10), 12741285.

6. Dadsetan, A., Khorsandi, A., Ahmadi, A., \& Mousavi, m. (2017). Investigation quantity and quality of Bojnourd municipal waste. The 4th International Conference on Environmental planning \& Management. Iran. [In Persian]

7. Den Boer, E., Jędrczak, A., Kowalski, Z., Kulczycka, J., \& Szpadt, R. (2010). A review of municipal solid waste composition and quantities in Poland. Waste Management, 30(3), 369-377.

8. Department of environmental health engineering, Yasouj university of medical science. (2012). Yasouj city integrated waste management. [In Persian]

9. Environmental research center of Tehran university of medical sciences. (2010). Qazvin Province integrated waste management. [In Persian]

10. Fan avari behmand Consulting Engineers Co. (2007). Bandar abbas integrated waste management. [In Persian]

11. Guerrero, L. A., Maas, G., \& Hogland, W. (2013). Solid waste management challenges for cities in developing countries. Waste management, 33(1), 220-232.

12. Hassanvand, M. S., Nabizadeh, R., \& Heidari, M. (2008). Municipal solid waste analysis in Iran. Iranian Journal of Health and Environment, 1(1), 9-18. [In Persian]

13. Hajinezhad, A., \& Servati, P. (2015). Bojnourd landfill leachate management by designing a new landfill using GIS and energy production by anaerobic digester. 2nd International Conference On IEA (Technology and Energy Management). [In Persian]

14. Heydari, M., \& Kanooni, R. (2013). Investigating the Impact of Solid Waste Recycling on the Urban Environment: A Case Study of Sanandaj. National conference of Geography, Urban Planning and Sustainable Development.

15. Hoornweg, D., \& Bhada-Tata, P. (2012). What a waste: a global review of solid waste management.

16. Hosseini, H., Zarifi Kliyani, S., \& Nezam pur, S. (2013). Investigation municipal and hospital waste in Karaj. International Conference on Civil Engineering Architecture \& Urban Sustainable Development $18 \& 19$ December 2013, Tabriz, Iran. [In Persian]

17. Idehpardazan tousei Consulting Engineers Co. (2012). Yazd integrated waste management. [In Persian]

18. Isfahan Municipality. (2014). Isfahan integrated waste management. [In Persian]

19. Jalilvand, R., Soluki, H.R., \& Hafezi Moghaddas, N. (2013). Assessing the suitability of landfills using the Ulkeno Index (Case study: Zahedan city). First National Conference on Planning for Conservation, Environmental Protection and Sustainable Development. [In Persian]

20. Karak, T., Bhagat, R. M., \& Bhattacharyya, P. (2012). Municipal solid waste generation, composition, and management: the world scenario. Critical Reviews in Environmental Science and Technology, 42(15), 1509-1630.

21. Kaza, S., Yao, Lisa C., Bhada-Tata, P., Van Woerden, F. (2018). What a Waste 2.0. A Global Snapshot of Solid Waste Management to 2050. Urban Development; Washington, DC: World Bank. World Bank. https://openknowledge.worldbank.org/handle/10986/30317 License: CC BY 3.0 IGO

22. Mahdavi Damghani, A. M., Savarypour, Gh., Zand, E., \& Deihimfard, R. (2008). Municipal solid waste management in Tehran: Current practices, opportunities and challenges. Waste management, 28(5), 929-934.

23. Maleki, A., Omrani, GH. (2002). Charactristics of Municipal Solid waste and Its Management in Hamedan. Yafteh , Volume 4 , Number 12; Page(s) 43-48. [In Persian] 
24. Manaf, L. A., Samah, M. A. A., \& Zukki, N. I. M. (2009). Municipal solid waste management in Malaysia: Practices and challenges. Waste management, 29(11), 2902-2906.

25. Mohammadi golrang, A., Hosseini, s., \& Dustdar, s. (2011). Destructive effect of the largest landfill leachate in north of Iran on the soil. The 5th national Conference \& Exhibition on Environmental Engineering, Tehran. [In Persian]

26. Naseri, N., Alighadri, M., Alipanah, A., Amiri, V., \& Mobasher Moghaddam, E. (2015). Urban waste disposal management (case study: Ardabil) the first national conference on environmental sciences and management in Iran, Ardabil. [In Persian]

27. National statistical yearbook, (2016). State Management and Planning Organization, Statistical Center of Iran. [In Persian]

28. Omrani, Gh. A. (2007). Principles and foundations of a sustainable strategy in waste management in Iran. The Third National Conference on Waste Management in Iran. [In Persian]

29. Pars amayesh Consulting Engineers Co. (2010). arak integrated waste management. [In Persian]

30. Ramavandi, B., Behrouzi, H., Parniani, N. (2014). Investigation of the potential and challenges of development of solid waste recycling in Bushehr. psj. 2014; 12 (2) :28-36 [In Persian]

31. Rastegar, A., Ghasemi, L., Abadi, A., \& Farzadkia, M. (2015). Investigating the amount of solid waste produced in Mashhad per year. Journal of Sabzevar University of Medical Sciences. Volume 22 / Number 6. [In Persian]

32. Ravanbakhsh, M., Abedinzadeh, N., \& Kazemi Rad, I. (2008). Solid waste disposal methods and their environmental problems: A case study of Rasht. Third National Congress on Recycling and Utilization of Renewable Organic Resources in Agriculture. Esfahan. [In Persian]

33. Sabz Andish Payesh Consulting Engineers Co. (2010 a). Tabriz integrated waste management. [In Persian]

34. Sabz Andish Payesh Consulting Engineers Co. (2010 b). golestan integrated waste management. [In Persian]

35. Sabz Andish Payesh Consulting Engineers Co. (2010c) lorestan integrated waste management. [In Persian]

36. Sabz Andish Payesh Consulting Engineers Co. (2020) Tehran integrated waste management. [In Persian]

37. Safari, Kh., Monavari, S.M., \& Abedi, Z. (2011). A review to Waste recycling cost-benefit analysis in Zahedan City. [In Persian]

38. Sanat pouyan sabz kavir Consulting Engineers Co. (2012). shahre kourd integrated waste management. [In Persian]

39. Sari factualy of natural resources-mazandaran recycling institute. (2010). Sari integrated waste management. [In Persian]

40. Saz ab pardazan Consulting Engineers Co. (2006). Ahvaz integrated waste management. [In Persian]

41. Semnan university Environmental Research Institute. (2016). Semnan integrated waste management. [In Persian]

42. Sharholy, M., Ahmad, K., Mahmood, G., \& Trivedi, R. C. (2008). Municipal solid waste management in Indian cities-A review. Waste management, 28(2), 459-467.

43. Shiraz university. (2015). Fars province integrated waste management. [In Persian]

44. Shokooh, A., Safari, E., \& Hashemi, S. H. (2013). Investigation of leachate quality from municipal waste landfill and compost plant (Case study: Mashhad). Environmental science and technology, Volume 15, Number 4. [In Persian]

45. Soleimanzadeh, Sh., Azizi, V., \& Abdi, E. (2013). Determining the economic benefits of recycling organic waste in Kermanshah. The 3th International Conference on Environmental planning \& Management. [In Persian]

46. Taghvayi, M., Mousavi, M., Kazemi Zad, Sh., \& Ghanbari, H. (2012). Municipal solid waste management, a step towards sustainable development. Case study: Zanjan. Journal of Urban and Regional Studies and Research, Year 3, Issue 12, Spring 2012. [In Persian]

47. Talaiekhozani, A., Nematzadeh, S., Eskandari, Z., Dehkordi, A. A., \& Rezania, S. (2017). Gaseous emissions of landfill and modeling of their dispersion in the atmosphere of Shahrekord, Iran. Urban Climate. [In Persian]

48. Taraghizadeh nazlou, P., \& Ganbarzadeh lak, M. (2015). Estimation of Extractable Energy from Urban Waste Landfills - Case Study: Urmia Urban Waste Landfill. Third National Conference on Environmental and Agricultural Research in Iran. [In Persian]

49. Tosie paydar shomalgan Consulting Engineers Co. (2006). Chatroud and Mahan districts of Kerman city integrated waste management. [In Persian]

50. Valizadeh, S., \& Shokri, Z. (2015). Application of Iranian Leopold Matrix in Environmental Impact Assessment (EIA) of Solid Waste Management Options in Birjand, Journal of Health and Environment, Iranian Journal of Environmental Health Scientific and Research Quarterly. Summer 2015, pages 224 to 262. [In Persian] 
51. Waste management act (2004). Islamic Consultative Assembly [In Persian]

52. Zhang, D. Q., Tan, S. K., \& Gersberg, R. M. (2010). Municipal solid waste management in China: status, problems and challenges. Journal of environmental management, 91(8), 1623-1633.

53. Zist aza mohit Consulting Engineers Co. (2014) llam integrated waste management. [In Persian]

54. Zurbrugg, Ch. (2002). Urban Solid Waste Management in Low - Income Countries of Asia: How to Cope with the Garbage Crisis. Department of Water and Sanitation in Developing Countries, Swiss Federal Institute for Environmental Science and Technology.

\section{Figures}

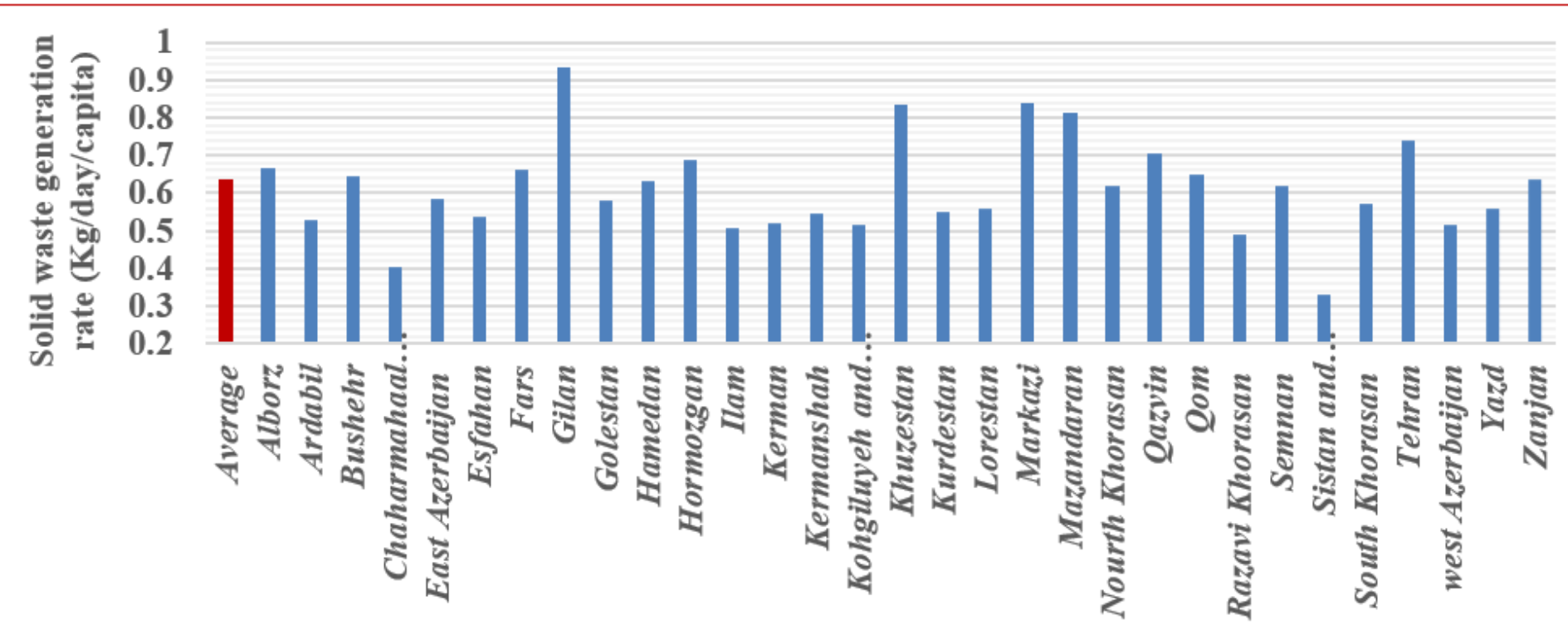

Province

Figure 1

Solid waste generation rate in Iran 


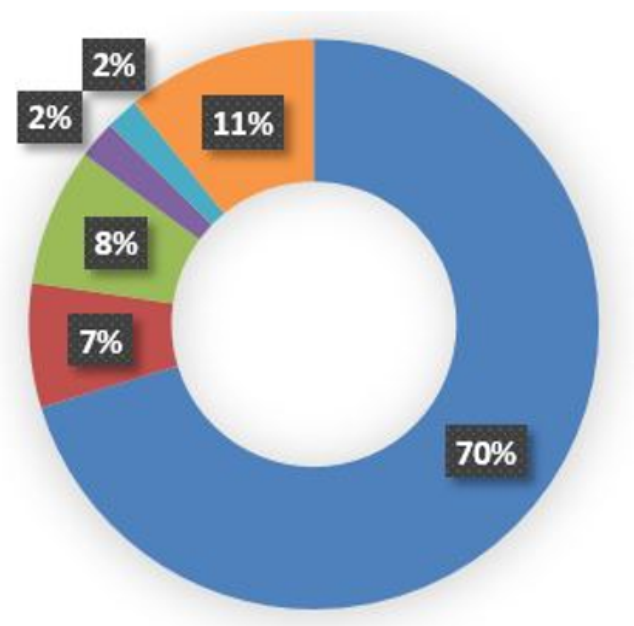

a) Iran MSW Composition

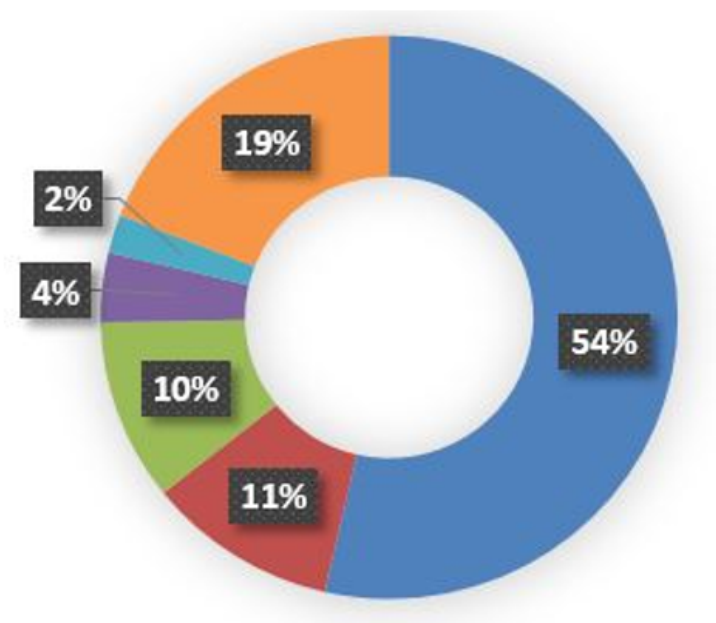

c) MSW composition in UMI countries

$$
\begin{aligned}
& \text { - Organic } \\
& - \text { Glass }
\end{aligned}
$$

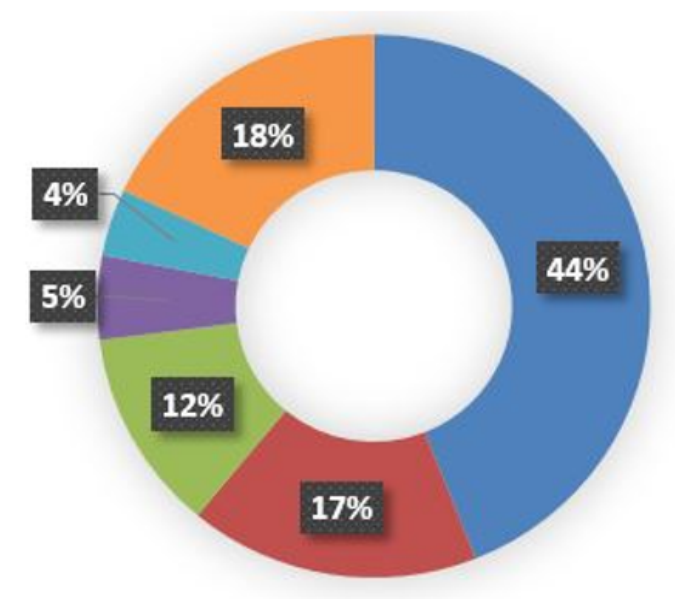

b) Worldwide MSW composition

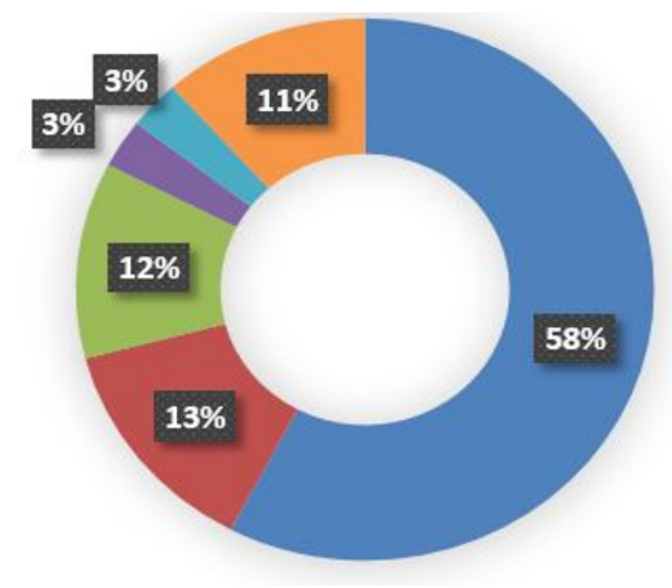

d) MSW composition in MENA countries

a Paper \& Cardboard E Plastic

anetals $\quad$ Others

\section{Figure 2}

MSW composition in a) Iran; b) World; c) Upper Middle Income (UMI) countries; and d) Middle East and North Africa (MENA) countries 


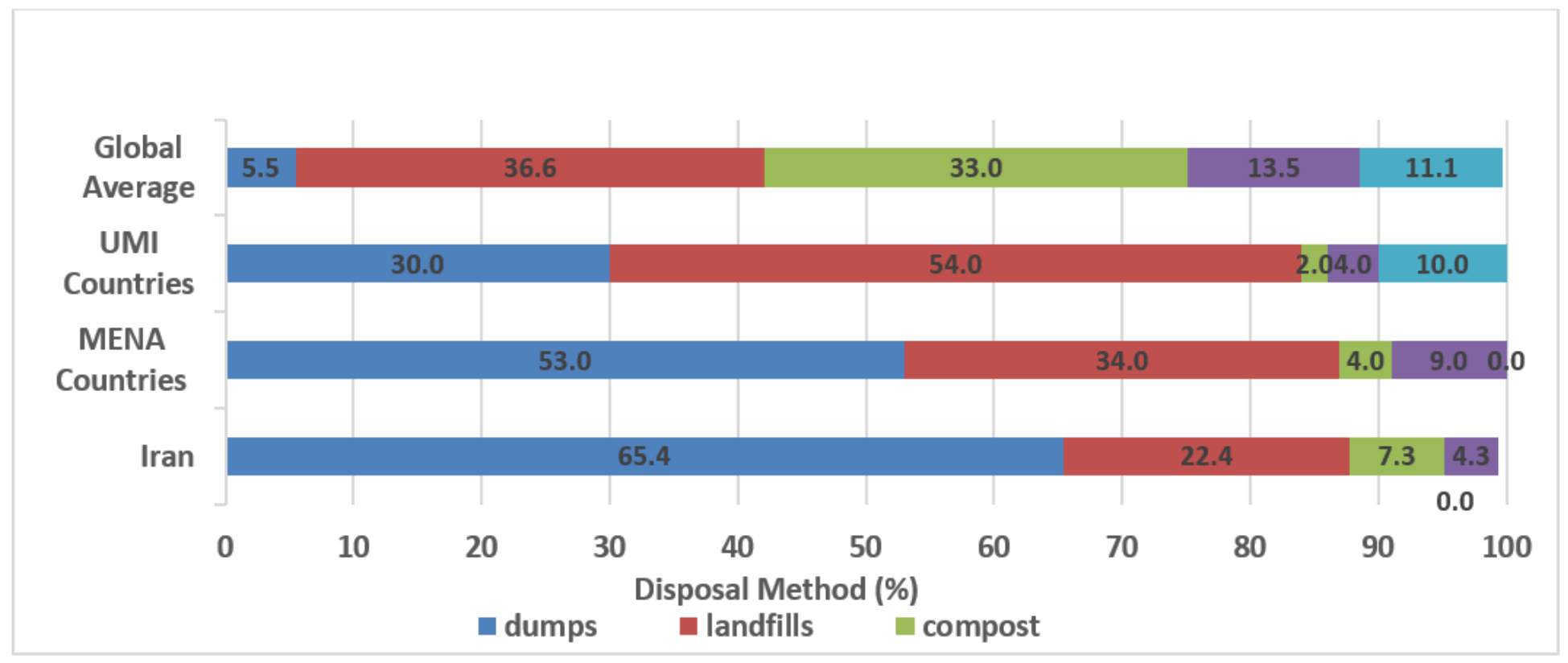

Figure 3

Solid waste disposal methods in Iran, UMI and MENA countries and the world 